\title{
On Optimal Size and Number of Reserves for Metapopulation Persistence
}

\author{
Rampal S. Etienne* and J. A. P. Heesterbeek \\ CBW-Centre for Biometry, P.O. Box 16, 6700 AA Wageningen, The Netherlands
}

(Received on 1 February 1999, Accepted in revised form on 24 November 1999)

\begin{abstract}
Habitat fragmentation is generally considered to be detrimental to the persistence of natural populations. In nature management, one therefore tends to prefer few large nature reserves over many small nature reserves having equal total area. This paper examines whether this preference is warranted in a metapopulation framework with circular reserves (patches) by formulating the dependence of metapopulation persistence on the size and number of reserves, both of which depend on reserve radius if the total area is kept constant. Two measures of metapopulation persistence are used: $R_{0}$, the number of patches colonized by an occupied patch during its lifetime as an occupied patch, and $T_{e}$, the expected time to extinction. These two measures are functions of the extinction and colonization rates of the metapopulation. Several mechanisms for the extinction and colonization processes are formulated from which the dependence of these rates on reserve radius is calculated. It turns out that $T_{e}$ generally increases with reserve radius for all mechanisms, which supports the preference of few large reserves. However, $R_{0}$ supports this preference only in the case of some special, rather unrealistic, mechanisms. In many other, more realistic, cases an intermediate reserve size exists for which metapopulation persistence measured by $R_{0}$ is optimal.
\end{abstract}

(C) 2000 Academic Press

\section{Introduction}

Habitat fragmentation is generally believed to be detrimental to the persistence of plant and animal populations (e.g. Forney \& Gilpin, 1989; Gonzalez et al., 1998). Indeed, there are several mechanisms by which species may suffer from habitat fragmentation, such as increased local extinction risk because of the larger impact of demographic and environmental stochasticity on smaller habitat patches; however, there are also (although fewer) mechanisms by which they may gain from it, such as recolonization of empty habitat patches by dispersers from still occupied patches (for more pros and cons of fragmentation see Burkey, 1996; Verboom et al., 1993 and the

\footnotetext{
* Author to whom correspondence should be addressed. E-mail: r.s.etienne@plant.wag-ur.nl
}

disussion). The cumulative effect of these opposing mechanisms should therefore be studied, but this has scarcely been done. Thus, in nature management one still faces the question whether or to what degree fragmentation is harmful. The (main aspect of this) problem can be restated more neutrally and more precisely in the following way: given a fixed amount of area $A_{\text {tot }}$ for nature reserves, what reserve size $A_{\text {res }}$ and hence what corresponding number $N_{\text {res }}=A_{\text {tot }} / A_{\text {res }}$ of reserves of this size are optimal for conservation purposes, that is, offer the best chances for the species to persist? This question is the central question in this paper where two different measures of persistence will be used $\left(R_{0}\right.$ and $T_{e}$; see the next section).

As stated, this question is similar to a problem commonly known as the SLOSS (single large or 
several small) problem, but contrary to the SLOSS problem, it allows intermediate solutions. To distinguish the question studied in this paper from the SLOSS problem and yet emphasize their close relationship, we will refer to the question in this paper as the FLOMS (few large or many small) problem.

This paper differs from most of the SLOSS literature in another respect as well. The SLOSS debate has been concerned mostly with the question of which reserve configuration provides the largest biodiversity, i.e. species richness (e.g. Quinn \& Harrison, 1988; Baz \& Garcia-Boyero, 1996); hence it is essentially a multi-species debate. This paper, however, focuses on single species for which the optimal configuration (size and number of reserves) needs to be determined. Stacey et al. (1997) come closer to the question posed in this paper because they compare few large populations with many small populations with the same total number of individuals using a single-species stochastic simulation model, and they conclude that if there is some connectivity an intermediate number of populations is optimal, that is, gives the longest mean time to extinction. However, keeping the total number of individuals constant is essentially different from fixing the total amount of reserve area.

We study the FLOMS problem here in a metapopulation setting with local extinctions in and recolonizations of identical circular patches in a lattice, which seems the most minimal setting in which the FLOMS problem makes sense. The aim of this paper is to explore several mechanisms for extinction and colonization in order to see how they scale with patch radius $R$, and hence to examine how patch radius affects the persistence of the metapopulation, in Hanski's (1991) words, to study their "compensatory effects".

We will discuss the implications and limitations of this approach for nature management at the end of the paper.

\section{Methods}

METAPOPULATION MODEL ASSUMPTIONS AND MEASURES OF PERSISTENCE

The FLOMS problem can be stated as follows: is it better for metapopulation persistence to have a few large (FL) patches or many small (MS) patches, where the size and number of patches are such that the total habitable area $A_{h}$ is the same in both cases? Two aspects of this question deserve scrutiny. The first is: how can a fair comparison between FL and MS be made? The second is: what does "better for metapopulation persistence" mean?

As regards the first aspect, in comparing an MS scenario and an FL scenario the only difference between FL and MS should be a difference in scale. This means that the patch configuration (i.e. relative patch positions) must be the same in both cases. However, this does not completely specify the absolute patch positions. So another assumption is needed. It seems most natural to assume then that not only is the total habitatable area the same for MS and FL, but also that on the scale of several patches the habitable area density is the same. Therefore, a sufficiently large number of patches (for the MS case and the FL case) is required to meet this criterion.

To facilitate computations, we will make the following assumptions which will be discussed later on:

- All patches are identical (in size, shape, quality, etc.) and they are circular with radius $R$.

- The patches form a regular lattice with each patch having $n$ nearest neighbors at equal center-to-center distance $L$. We will present results for a hexagonal lattice $(n=6$; see Fig. 1) and a square lattice $(n=4)$. For other values of $n$ some modifications of the formulae may be needed.

Figure 1 shows two scenarios with a hexagonal lattice of circular patches: one with a few large patches (lattice distance $L_{F L}$, patch radius $R_{F L}$ ) and one with many small patches (lattice distance $L_{M S}=\frac{1}{2} L_{F L}$, patch radius $R_{M S}=\frac{1}{2} R_{F L}$ ), but both with $R / L=\frac{1}{4}$. In this setting, the scale parameter which differs between the FL and MS cases is the patch radius $R$. The FL and MS cases have equal area density because one can show that the total habitable area inside the dotted rectangle is given by $(16 \pi / 8 \sqrt{3}) R^{2} / L^{2}$ in both cases. If one includes patches at the edges in full, the total habitable area is not exactly the same for FL and MS. 


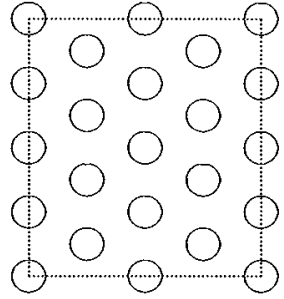

Few large

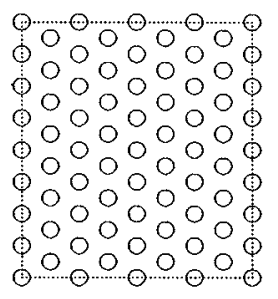

Many small
FIG. 1. The FLOMS-problem for a hexagonal patch configuration: which configuration is optimal for metapopulation persistence: few large patches (FL) or many small patches (MS)? Here $R_{M S}=\frac{1}{2} R_{F L}$, but in both cases $L=4 R$. This implies that the amount of patch area within the dotted rectangle is the same in both cases. The area outside the dotted rectangle is not the same in both cases, but this difference will become smaller for a larger number of patches inside the dotted rectangle.

Therefore, we assume that the number of patches is sufficiently large to make these edge effects negligibly small.

Let us move on to the second aspect of the question above: what does "better for metapopulation persistence" mean? The word "persistence" is used here in its informal, nonmathematical sense which is approximately equivalent to "survival". To answer the question we employ two measures of metapopulation persistence; "better for metapopulation persistence" then means a higher value of these measures.

The first measure is the expected time required by the metapopulation to reach extinction. This expected time to metapopulation extinction will be denoted by $T_{e}^{\text {meta }}$ or just $T_{e}$. Because larger patches have larger interpatch distances (see Fig. 1), the probability that a patch will be able to colonize another patch will become smaller with increasing patch size, and the expected extinction time $T_{e}$ of the metapopulation will become an increasing function of the expected time to local extinction $T_{e}^{\text {local }}$ :

$$
T_{e}:=T_{e}^{\text {meta }} \sim T_{e}^{\text {local }} \text { for large } R .
$$

As a second measure of metapopulation persistence we use the basic reproduction ratio $R_{0}$ which can be interpreted as the colonization potential of an occupied patch. More precisely, it is the average number of successful dispersers produced by a single occupied patch before its extinction, successful meaning that the dispersers reach a new (empty or occupied) patch and settle there. In short, $R_{0}$ is the number of colonizations from a single occupied patch. We call it a colonization potential because it bears some similarities to the population potential (Pooler, 1987). $R_{0}$ reflects the capacity of the metapopulation to return to its original configuration after a disturbance to a very low occupation level. The benefits and limitations of $R_{0}$ as a measure of metapopulation persistence will be discussed later.

Suppose that a patch with local extinction time $T_{e}^{\text {local }}$ colonizes other patches at a constant rate $c$. Then $R_{0}$ equals the product of $c$ and $T_{e}^{\text {local }}$ :

$$
R_{0}=c T_{e}^{\text {local }} .
$$

For the lattices studied here, $R_{0}$ is the same for every patch (ignoring edge effects), so the colonization potential of the entire metapopulation can be represented by the $R_{0}$ of a single patch.

The FLOMS problem can now be stated more precisely as the question of how the measures of persistence, $T_{e}$ and $R_{0}$ depend on patch radius $R$. Therefore, we will formulate several plausible mechanisms for $T_{e}^{\text {local }}$ and $c$, and derive expressions for these parameters as a function of patch radius $R$. Since the objective is only a comparison of $R_{0}$ and $T_{e}$ for the FL and the MS cases, it suffices to know how the colonization and extinction parameters scale with $R$, i.e. proportionality constants are irrelevant.

We will derive these expressions for the hexagonal and square patch configurations described above using some assumptions which simplify computations, and present the results for this simple case. Then we will discuss how the results may be affected when these assumptions are relaxed. The assumptions, in addition to the two assumptions above, are the following.

- There is no influence of dispersal on local population size (neither by emigration, nor by immigration; see however the remark made in the section about dispersal after depletion).

- Spatial correlation of local extinction times plays no role in metapopulation persistence.

- The colonization rate is constant and can be split into $c_{\text {out }}$ and $c_{i n}$ :

$$
c=c_{\text {out }} c_{\text {in }}
$$


where the parameter $c_{\text {out }}$ is the emigration rate (the number of dispersers produced per unit time) per occupied patch, and the quantity $c_{i n}$ is the probability that a disperser reaches a patch and settles successfully. Then

$$
\frac{R_{0}}{c_{\text {in }}}=c_{\text {out }} T_{e}^{\text {local }}
$$

is the number of dispersers produced by an occupied patch during its lifetime as an occupied patch, and

$$
R_{0}=c_{\text {out }} c_{\text {in }} T_{e}^{\text {local }}
$$

is the fraction of those dispersers eventually settling successfully.

- Local populations are almost always at carrying capacity which is an increasing power function of $R$. More precisely, we will assume that populations grow quickly to the carrying capacity $K$, the maximum number of individuals a patch can sustain, and remain there most of the time. We will assume that $K \geqslant 1$, and that $K$ scales linearly with area, that is,

$$
K=C_{1} R^{2} .
$$

where $C_{1}:=1 / R_{\min }^{2}$ with $R_{\text {min }}$ being the minimum patch radius to sustain one individual (here after $C_{i}$, with $i$ any integer, will denote a species-specific and/or system-specific constant). Equation (6) is supported both theoretically and empirically (Hanski, 1997; Kindvall \& Ahlén, 1992, and references therein). However, other empirical data do not display any significant relationship with $R$ (Kindvall \& Ahlén, 1992, and references therein; Manne et al., 1998), while Hanski et al. (1996) find that

$$
K \sim R
$$

Nevertheless, it will turn out that any positive power of $R$ will suffice to reach the same qualitative conclusions.

\section{Mechanisms for $T_{e}^{\text {local }}$}

Local extinction can be due to demographic and environmental stochasticity, catastrophes and genetic processes such as random drift and inbreeding depression (Schaffer, 1981). Here only demographic and environmental stochasticity are studied, although the impact of catastrophes (Spiller et al., 1998; Wardle et al., 1997) and genetic processes (Boecklen, 1986; Frankham \& Ralls, 1998; Saccheri et al., 1998) can obviously play a role as well.

\section{DEMOGRAPHIC STOCHASTICITY}

Using a stochastic differential equation with only demographic stochasticity, Foley (1997) derives the following dependence of the expected time to local extinction $T_{e}^{\text {local }}$ on patch radius $R$ :

$$
T_{e}^{\text {local }}(R) \sim \mathrm{e}^{\varepsilon R^{2}}
$$

with $\varepsilon^{-1 / 2}$ the characteristic radius of extinction. Foley (1997) finds that $\varepsilon$ is proportional to $\alpha / \operatorname{Var}\left(\alpha_{1}\right)$. Here $\alpha$ is the (mean) net overall reproduction rate, and $\alpha_{1}$ is the (mean) net reproduction rate for one individual; $\operatorname{Var}(\alpha)=\operatorname{Var}\left(\alpha_{1}\right) / N$, where $N$ is the number of individuals. Equation (8) is valid as long as $\left[\alpha / \operatorname{Var}\left(\alpha_{1}\right)\right] \ln K$ is sufficiently large, $K$ being the carrying capacity.

\section{ENVIRONMENTAL STOCHASTICITY}

Using a stochastic differential equation with environmental stochasticity, Foley (1997) derives

$$
\begin{aligned}
T_{e}^{\text {local }}(R) \propto \mathrm{e}^{2(\alpha / \operatorname{Var}(\alpha)) \ln C_{1} R^{2}} & \\
& -1-2 \frac{\alpha}{\operatorname{Var}(\alpha)} \ln C_{1} R^{2}
\end{aligned}
$$

with $\alpha$ again being the net overall reproduction rate.

If $[|\alpha| / \operatorname{Var}(\alpha)] \ln C_{1} R^{2}$ is large and $\alpha$ negative, then eqn (9) approaches

$$
T_{e}^{\text {local }}(R) \propto \ln C_{1} R^{2} .
$$

For small $[|\alpha| / \operatorname{Var}(\alpha)] \ln C_{1} R^{2}$ with $\alpha$ positive or negative, eqn (9) reduces to (see also Goodman, 1987)

$$
T_{e}^{l o c a l}(R) \propto\left(\ln C_{1} R^{2}\right)^{2}
$$

which entails that $R_{\min }$ is indeed a threshold. 

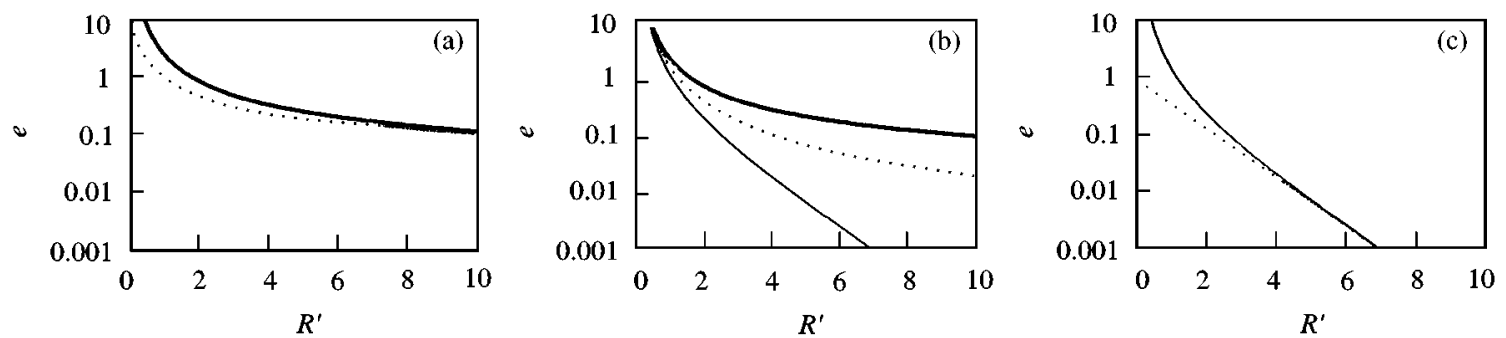

FIG. 2. Graphs of the extinction rate $e:=1 / T_{e}^{1 / l o c a l}$ as a function of the rescaled patch radius $R^{\prime}:=2|\alpha| / \operatorname{Var}(\alpha), \ln C_{1} R^{2}$ for environmental stochasticity. The solid curve represents the full relationship of eqn (9) for negative $\alpha$ (thick curve) and positive $\alpha$ (thin curve). The dotted curves are the approximations: (a) (—) eqn (9), $\alpha<0 ;(\cdots \cdots)$ eqn (10). (b) $(-)$ eqn (9), $\alpha<0 ;(-)$ eqn (9), $\alpha>0 ;(\cdots)$ ) eqn (11). (c) (-) eqn (9), $\alpha>0 ;(\cdots)$ eqn (12).

For large $[|\alpha| / \operatorname{Var}(\alpha)] \ln C_{1} R^{2}$ with $\alpha$ positive, eqn (9) behaves according to

$$
T_{e}^{\text {local }}(R) \propto R^{\mu}
$$

with $\mu=4(\alpha / \operatorname{Var}(\alpha))$; see also Fig. 2. Foley (1997) gives estimations of $\alpha / \operatorname{Var}(\alpha)$ which are usually between 0 and 1 , but occasionally just below zero or very large.

Assuming environmental stochasticity but without a stochastic differential equation, Allen et al. (1992) also derive eqn (12) where $\mu$ ranges between 1 and 2. Hanski uses eqn (12) and reports $\mu$-values of $0.92,1.82,4.60$ for shrews (Hanski, 1997) and 1.002, 2.118, 1.580 for butterflies (Hanski, 1992). Using the population viability analysis package ALEX (Possingham et al., 1992; Possingham \& Davies, 1995), Day \& Possingham (1995) report a relationship similar to eqn (12) with $\mu=1$.

With respect to the joint effect of both environmental and demographic stochasticity, Foley (1997) notes that adding demographic stochasticity to environmental stochasticity hardly affects the dependence of $T_{e}^{\text {local }}(R)$ on $R$. This is due to the marginal effect of demographic stochasticity when population number is high.

\section{Mechanisms for $c_{\text {out }}(R)$}

For the dispersal rate from a patch $c_{\text {out }}(R)$, several mechanisms can be imagined, such as dispersal due to the avoidance of competition, a constant fraction of dispersers or resource depletion. A more phenomenological approach uses a maximum distance of dispersal. Let us take a closer look at these options.

\section{AVOIDING COMPETITION}

Assume that the population is at carrying capacity where any excess reproduction does not vanish because of competition as in the logistic growth model, but it vanishes because of dispersal to avoid competition (Hansson, 1991). This may be valid for territorial species. If the net reproduction rate is again denoted by $\alpha$, then $c_{\text {out }}(R)$ is given by

$$
c_{\text {out }}(R)=K \alpha \propto R^{2}
$$

again assuming that eqn (6) holds.

\section{CONSTANT FRACTION OF DISPERSERS}

If a constant fraction $\varphi$ of the population disperses (e.g. juveniles leave a patch at the end of the season), and the population is assumed to be at carrying capacity which obeys eqn (6), then

$$
c_{\text {out }}(R)=K \varphi \propto R^{2}
$$

which is similar to eqn (13).

\section{DISPERSAL AFTER DEPLETION}

If a patch is left only when the resources are depleted (Bell, 1991; Hansson, 1991), then for dispersal to come about, the resource recovery rate must be lower than its depletion rate. If this is indeed the case, then the dispersal rate is proportional to the number of organisms present 
in the patch, which itself is presumably proportional to this resource threshold and hence to area by assumption. Therefore,

$$
c_{\text {out }}(R) \propto R^{2}
$$

which is similar to eqns (13) and (14). If the patch is left by (almost) all organisms at the same time, then extinction and colonization become correlated, which alters expression (2) to $R_{0}=K c_{i n} \propto R^{2} c_{i n}$ which will turn out not to affect the results of this paper qualitatively. However, this correlation between extinction and colonization may cause extinctions between patches to become correlated; in that case one of the assumptions above is violated, which may well affect the results of this paper.

\section{MAXIMUM DISTANCE OF DISPERSAL}

Another approach, suggested by Vos et al. (1999), is based on the assumption that dispersers are only produced in an outer strip or ring of the patch. If $R_{s}$ is the width of the outer strip of each circular patch, then $c_{\text {out }}(R)$ is proportional to the number of organisms living in this strip, and hence to the area of this strip (assuming e.g. constant density within the strip), which is $\pi R^{2}-\pi\left(R-R_{s}\right)^{2}$. Then,

$c_{\text {out }}(R)= \begin{cases}C_{2} R R_{s}\left(2-\frac{R_{s}}{R}\right) & \text { for } R \geqslant R_{s}, \\ C_{2} R^{2} & \text { for } R \leqslant R_{s},\end{cases}$

where $C_{2}$ is a constant. So, assuming $R \geqslant R_{s}$, $c_{\text {out }}(R) \propto R$. This linear behavior with a threshold is qualitatively similar to diffusion with logistic growth. Indeed, in the latter case one can show that the density of organisms is at the carrying capacity, except for a strip near the edge of the patch, which has an approximately constant width. Equation (16) seems to be supported by Woodroffe \& Ginsberg (1998), who remark that patch edge size rather than population size determines the dispersal rate.

\section{Mechanisms for $c_{i n}(R)$}

The probability that a disperser arrives at any patch conditional on leaving a certain patch, $c_{i n}(R)$, involves at least three factors: the behavior (movement pattern, velocity) of the disperser, the risk the disperser runs while dispersing and the settlement process (patch selection strategy). As far as the first factor is concerned, we will assume that the disperser moves at a constant speed. Two movement mechanisms will be considered: straight walk and random walk. With regard to the second factor, we will assume that there is a constant mortality rate $\lambda$. As for the third factor, for each of the two movement mechanisms one or two patch selection strategies will be chosen.

\section{STRAIGHT WALK}

Straight walk is defined as movement at a constant velocity $v$ (constant in magnitude and direction). The patch selection strategies to be discussed are nearest-neighbor dispersal, the "settlement-death" strategy, adapted from Vos et al. (1999) and the "pie-slice" strategy, implicit in Lindenmayer \& Possingham (1995). For both strategies we have in a patch configuration with $n(=4,6)$ nearest neigbors

$$
c_{\text {in }}(R, n)=\sum_{i} p_{\text {survival }}\left[d_{i}(R)\right] p_{\text {settlement }}\left[d_{i}(R), n\right]
$$

where $p_{\text {survival }}\left(d_{i}\right)$ is the probability of surviving distance $d_{i}$ to patch $i$ (center-to-center distance), which is given by

$$
p_{\text {survival }}\left(d_{i}\right)=\mathrm{e}^{-\lambda_{1}\left(d_{i}-(3 / 2) R\right)}
$$

and where $p_{\text {settlement }}\left(d_{i}, n\right)$ is the probability of settlement at distance $d_{i}$, in which the two patch selection strategies differ. Here $i$ refers to any patch surrounding the patch abandoned by the disperser, and $\lambda_{1}$ is the mortality rate per unit distance: $\lambda_{1}=\lambda / v$. One can interpret $\lambda_{1}^{-1}$ as the average distance traveled before dying. Expression (18) for $p_{\text {survival }}\left(d_{i}\right)$ is just the survival function with hazard function $\lambda\left(t=d_{i} / v\right)=\lambda$; the presence of $\mathrm{e}^{(3 / 2) \lambda_{1} R}$ in eqn (18) is due to the fact that part of the trip of distance $d_{i}$ lies within either the patch of origin $(R)$ or the patch of destination (approximately $(1 / 2) R$; in fact it is a lower bound); intermediate patches are not considered. 
Straight Walk and Nearest-Neighbor Dispersal

For nearest-neighbor dispersal, $p_{\text {settlement }}$ is simply

$p_{\text {settlement }}\left(d_{i}, n\right)$

$=\left\{\begin{array}{l}\frac{1}{n} \text { if patch } i \text { is a nearest neighbor, } \\ 0 \text { for all other patches. }\end{array}\right.$

With lattice distance $L \equiv h R, h$ being a constant reflecting the total area density which is the same in the FL and MS cases, we arrive at

$$
c_{i n}(R, n)=\mathrm{e}^{-\lambda_{1} R(h-3 / 2)} .
$$

Straight Walk and the Settlement-death Strategy

The settlement-death strategy assumes that dispersers prefer nearby patches over distant patches, and large patches over small patches. Mathematically,

$$
p_{\text {settlement }}\left(d_{i}, n\right)=\frac{A_{i} \mathrm{e}^{-\lambda_{2} d_{i}}}{\sum_{i} A_{i} \mathrm{e}^{-\lambda_{2} d_{i}}}
$$

where $A_{i}$ is the size of patch $i$ which will drop out from the equation because all patches are identical, and $\lambda_{2}$ is the constant settlement rate per unit distance. $\lambda_{2}^{-1}$ can be interpreted as the average distance an individual wants to travel. Evidently $\lambda_{2}^{-1}$ may depend on $\lambda_{1}^{-1}$ : the willingness of a mouse to cross a large open grass land may be much smaller than its willingness to walk from stepping stone to stepping stone which is presumably safer. In eqn (21) the normalization stems from the assumption that without death settlement will always occur. Although eqn (18) is often used (e.g. MacArthur \& Wilson, 1967; Hanski, 1997, where $\lambda_{1}$ is called $\alpha$ ) and will also be used here, it should be noted that Hill et al. (1996) show some empirical data supporting a power function, i.e. $p_{\text {survival }}\left(d_{i}, n\right) \sim d_{i}^{-\chi}$ where $\chi$ is a positive parameter, while Gilpin \& Diamond (1976) study various values for the parameter $y$ in the expression $p_{\text {survival }}\left(d_{i}\right) \sim \mathrm{e}^{-d_{0}^{-1} d_{i}^{v}}, d_{0}$ being a constant and find that $y=0.553$ fits their data best. However, neither Hill et al. (1996) nor Gilpin \& Diamond (1976) provide an explanatory mechanism. With again lattice distance $L \equiv h R$, $c_{i n}(R, n)$ turns into

$$
\begin{aligned}
& c_{i n}(R, n)= \\
& \begin{array}{l}
\mathrm{e}^{(3 / 2) \lambda_{1} R}\left(\frac{\lambda_{2}}{\lambda_{1}+\lambda_{2}}\right)^{2} \\
\quad \text { or }\left(\lambda_{1}+\lambda_{2}\right) R h \ll 1 \\
\frac{n\left(\lambda_{2} R h\right)^{2} \sin 2 \pi / n}{2 \pi} \mathrm{e}^{-\lambda_{1} R(h-(3 / 2))-\lambda_{2} R h} \\
\text { for } \lambda_{1} R h \gg 1 \text { and } \lambda_{2} R h \ll 1\left(\text { so } \frac{\lambda_{2}}{\lambda_{1}} \ll 1\right) \\
\mathrm{e}^{-\lambda_{1} R(h-(3 / 2))} \\
\text { for } \lambda_{2} R h \gg 1 .
\end{array}
\end{aligned}
$$

See Appendix A for details of the derivation of these approximations.

\section{Straight Walk and the Pie-slice Strategy}

The pie-slice strategy is more spatially explicit. It assumes that dispersers choose a patch to disperse to with a probability proportional to its size as it appears on the horizon of the disperser. This leads to

$$
p_{\text {settlement }}\left(d_{i}, n\right)=\frac{f_{i} \Delta \varphi_{i}(R, n)}{2 \pi} .
$$

Here, $\Delta \varphi_{i}$ is the angle of the horizon covered by patch $i$, and $f_{i}$ is the fraction of the patch that is observable (i.e. not blocked from view by another patch) by the disperser from its patch of origin.

One can show (see Appendix A) that the following asymptotic expression for $c_{i n}(R, n)$ holds:

$$
\begin{aligned}
c_{i n}(R, n) \approx & \frac{n}{\pi} \mathrm{e}^{-\lambda_{1} R(h-3 / 2)} \arccos \left[1-\frac{1}{2 h^{2}}\right] \\
& \text { for } \lambda_{1} R h \gg 1
\end{aligned}
$$

No such approximation is possible for small $\lambda_{1} R h$. 


\section{RANDOM WALK}

Random walk (i.e. diffusion) can also be used as an approach to $c_{i n}(R)$ (see Appendix B). One may use the solution to the stationary $(\partial x / \partial t=0)$ diffusion equation with a sink term $\sigma=-\lambda x$, where $x$ is disperser density and $\lambda$ again denotes the death rate. Let $D$ be the diffusion coefficient outside the patches, and define $\lambda_{1}:=\sqrt{\lambda / D}$ which may be interpreted as the mortality rate per unit distance dispersed. $c_{i n}(R)$ is here defined as the quotient of the rate of dispersers entering destination patches and the rate of dispersers leaving the patch of origin, which is $c_{\text {out }}(R)$ :

$$
c_{\text {in }}(R)=\frac{\int_{\partial A_{\text {in }}}(D \nabla x, \mathrm{~d} \mathbf{A})}{c_{\text {out }}(R)}
$$

where $\partial A_{\text {in }}$ denotes the combined boundaries of the destination patches, and $(D \nabla x, \mathrm{~d} \mathbf{A})$ is the component of the gradient of the disperser density perpendicular to the destination patch boundary, i.e. the flux over the destination patch boundary. For the pie-slice strategy and sufficiently large $\lambda_{1} R h$, this reduces to (see Appendix B)

$$
\begin{aligned}
c_{i n}(R, n) \approx & n R \sqrt{h-\frac{1}{2}} \mathrm{e}^{-\lambda_{1} R(h-3 / 2)} \\
& \times \arccos \left[1-\frac{1}{2 h^{2}}\right] \text { for } \lambda_{1} R h \gg 1 .
\end{aligned}
$$

\section{Results: $T_{e}(R)$ and $R_{0}(R)$}

With all functions of eqn (2) having been defined, $T_{e}(R)$ and $R_{0}(R)$ can be calculated. Taking all suggested mechanisms for $c_{\text {out }}$ into account, one obtains

$$
c_{\text {out }}(R) \propto R^{\gamma}
$$

with $0 \leqslant \gamma \leqslant 2$. Likewise, all dispersal strategies for $c_{i n}(R)$ can be summarized as

$$
c_{i n}(R) \propto\left\{\begin{array}{cc}
\mathrm{e}^{-\lambda_{1} R\left(\beta h-h_{0}\right)} & \text { for } \lambda_{i} R h \ll 1 \\
R^{v} \mathrm{e}^{-\lambda_{1} R\left(h-h_{0}\right)} & \text { for } \lambda_{i} R h \gg 1
\end{array}\right.
$$

with $\beta=0$ or $1, v=0,1$ or 2 , and $h_{0}=1,3 / 2$, or $3 / 2-\left(\lambda_{2} / \lambda_{1}\right) h ; i$ stands for both 1 and 2 . If $\beta=1$, $v=0$. Now, with eqns (8), (10)-(12), (27) and (28), eqns (1) and (2) yield the following expressions for $T_{e}(R)$ and $R_{0}(R)$ :

$T_{e}(R) \sim T_{e}^{\text {local }}(R) \quad$ for large $R$

$$
\propto \begin{cases}\mathrm{e}^{\varepsilon R^{2}} & \left(\frac{\alpha}{[\operatorname{Var}(\alpha)]_{1}} K \gg 1\right), \\ R^{\mu} & \left(\frac{\alpha}{[\operatorname{Var}(\alpha)]} K \gg 1\right), \\ \ln C_{1} R^{2} & \left(\frac{-\alpha}{[\operatorname{Var}(\alpha)]} K \gg 1\right)\end{cases}
$$

and

$$
\begin{aligned}
& R_{0}(R)=c(R) T_{e}^{\text {local }}(R)
\end{aligned}
$$

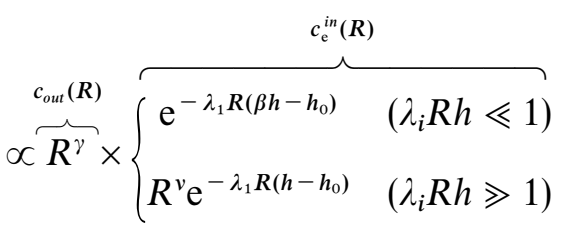

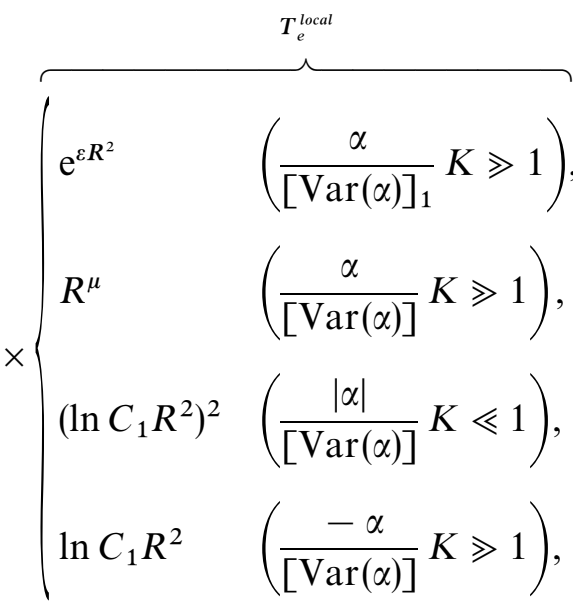

where in the $T_{e}^{\text {local }}$-term the first expression is valid for demographic stochasticity and the others are valid for environmental stochasticity, and $K=C_{1} R^{2}$.

It is easy to see that $T_{e}(R)$ increases with $R$ for large $R$, so using this measure of metapopulation 
persistence supports the widely adopted view that habitat fragmentation is harmful. However, this is generally not true if $R_{0}$ is used as the measure of metapopulation persistence. Consider the case with only environmental stochasticity. If we make the assumptions that $\lambda_{1}>0$ and $\gamma+v>0$, then we see that there will be an optimal patch size if $R_{0}(R)$ is used as the measure of metapopulation persistence. As $R$ increases, $R_{0}(R)$ will sooner or later enter the region for which $\lambda_{i} R h \gg 1$, where the decreasing exponential term of $c_{i n}(R)$ dominates causing $R_{0}(R)$ to decrease with $R$ in that region. Mathematically,

$$
R_{0}(R) \propto f(R) R^{\gamma+v} \mathrm{e}^{-\lambda_{1} R\left(h-h_{0}\right)} \quad \text { if } \lambda_{i} R h \gg 1,
$$

where $f(R)$ is a function which increases with $R$, yet slower than an exponential function.

With only demographic stochasticity, and the same assumptions $\lambda_{1}>0$ and $\gamma+v>0$, we get

$$
R_{0}(R) \propto \mathrm{e}^{-\lambda_{1} R\left(h-h_{0}\right)+\varepsilon R^{2}} R^{\gamma+v} \quad \text { if } \lambda_{i} R h \gg 1 .
$$

It is not difficult to see that $R_{0}(R)$ will eventually increase monotonically with $R$, but for $\quad \lambda^{2}-8 \varepsilon(\gamma+v)>0, \quad \lambda:=\lambda_{1}\left(h-h_{0}\right)$ there are two extrema as Fig. 3 shows. This means that for demographic stochasticity, enlarging patch size may cause a (temporary) decrease in $R_{0}(R)$.

Before discussing the consequences of these findings, let us look at an example. Since in reality there is always some environmental stochasticity and the carrying capacity $K$ of the patch is not extremely small (otherwise we would not regard it as habitable), eqn (12) is the most widely applicable choice for $T_{e}^{\text {local }}$. Assuming straight walk and the settlement-death strategy [eqn (A.1)], we arrive at

$$
R_{0}(R) \propto R^{\gamma+\mu} \mathrm{e}^{(3 / 2) \lambda R} \frac{S_{1}\left(\lambda_{1}+\lambda_{2}\right)}{S_{1}\left(\lambda_{2}\right)}
$$

which is plotted in Fig. 4. The optimum value for $R_{0}(R)$ occurs when the derivative of $R_{0}(R)$ with

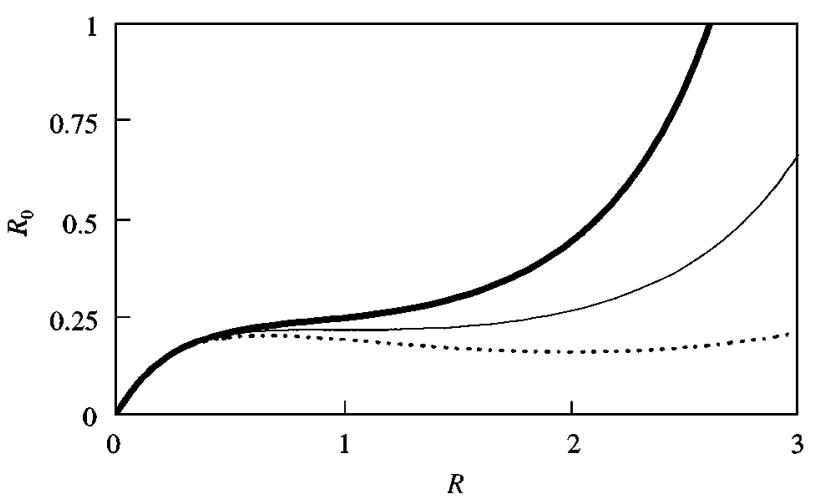

FIG. 3. Plots of $R_{0}(R)=C \mathrm{e}^{-\lambda R+\varepsilon R^{2}} R^{\gamma+v}$ vs. $R$ for several values of $\lambda^{2}-8 \varepsilon(\gamma+v)$, where $\gamma+v>0$, and $C=1$ : $(\cdots \cdots) \quad \lambda^{2}-\varepsilon(\gamma+v)>0 ; \quad(-) \quad \lambda^{2}-\varepsilon(\gamma+v)=0 ; \quad(-)$ $\lambda^{2}-\varepsilon(\gamma+v)<0$.

respect to $R$ equals 0 :

$$
\begin{aligned}
\frac{\mathrm{d} R_{0}(R)}{\mathrm{d} R} & =0 \Rightarrow \frac{\gamma+\mu}{R}+\frac{3}{2} \lambda_{1} \\
& =-\frac{\mathrm{d} \ln \left(S_{1}\left(\lambda_{1}+\lambda_{2}\right) / S_{1}\left(\lambda_{2}\right)\right)}{\mathrm{d} R}
\end{aligned}
$$

Using eqn (A.2), this leads to the following condition for the optimal radius $R_{\text {opt }}$ :

$$
\begin{aligned}
& \frac{R_{o p t}}{\lambda_{1}^{-1}} \geqslant \frac{\gamma+\mu}{h-3 / 2} \\
& \quad \text { for } \gamma+\mu>0 \text { and } \lambda_{2} R_{o p t} h \gg 1, \\
& R_{o p t}=0 \text { for } \gamma+\mu \leqslant 0 .
\end{aligned}
$$

This optimal radius can indeed be seen in the plots of $R_{0}(R)$ in Fig. 4. For the nearest-neighbor dispersal strategy, the equality sign in the first equation of eqn (35) applies.

\section{Discussion}

The results show that two different measures of metapopulation persistence lead to two different opinions on the FLOMS problem if environmental stochasticity with or without demographic stochasticity is assumed. On the one hand, someone who wants to maximize the colonization potential $R_{0}$ will advocate an intermediate 

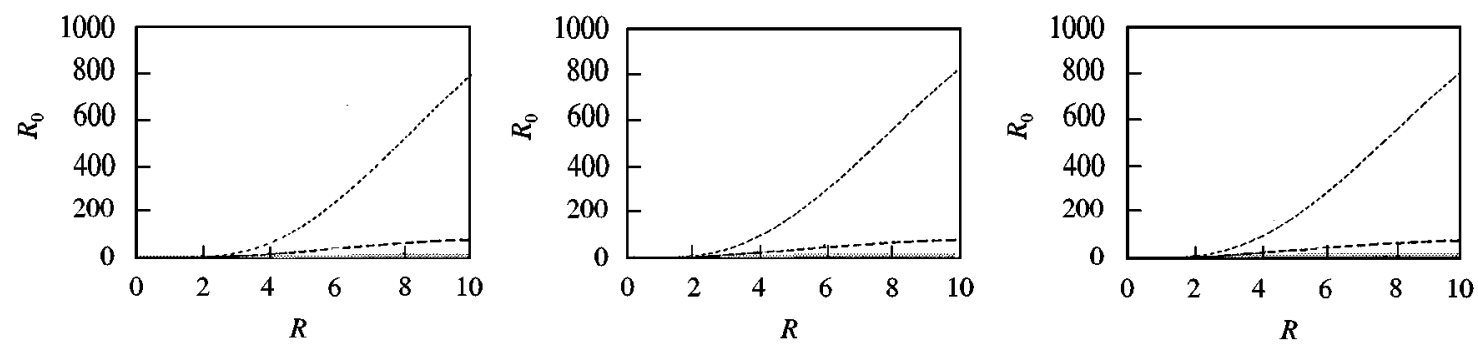

$\lambda_{1}=0.5 ; \lambda_{2}=0.25$
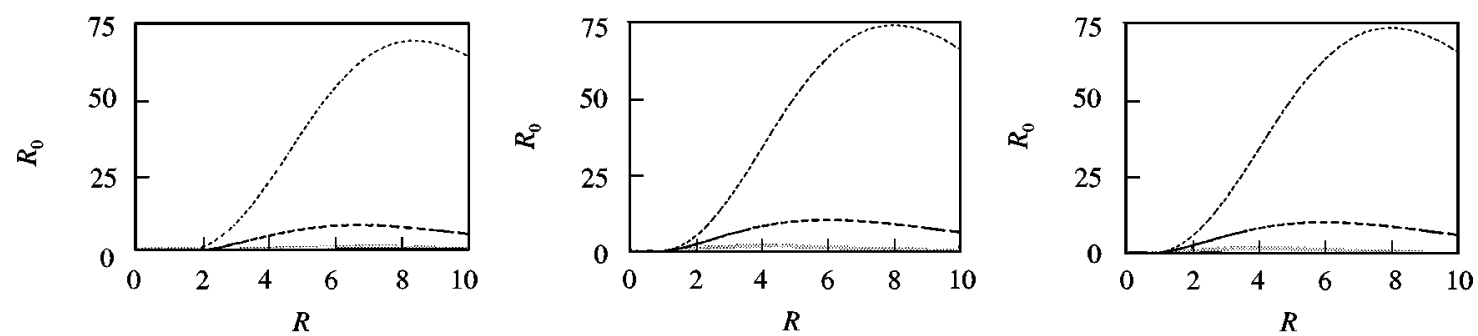

$\lambda_{1}=1 ; \lambda_{2}=0.25$

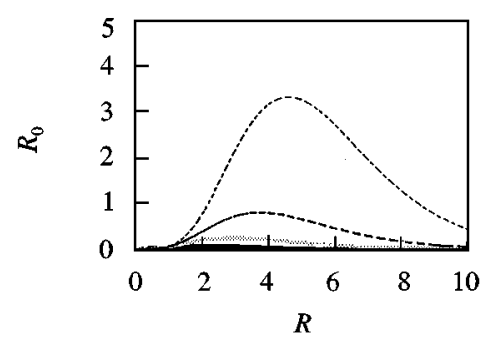

$\lambda_{1}=2 ; \lambda_{2}=0.25$
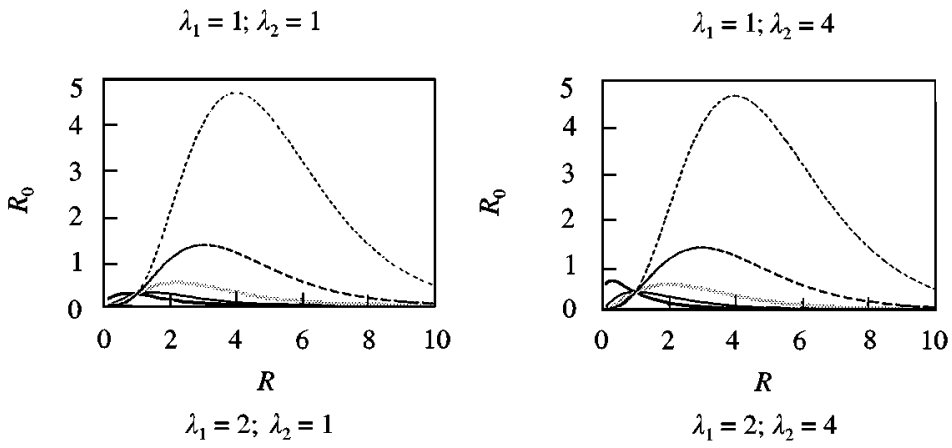

FIG. 4. Plots of $R_{0}(R)$ vs. $R$ using environmental stochasticity and the settlement-death strategy for different values of $\lambda_{1}$ and $\lambda_{2}$ and $k:=\gamma+\mu$ ( $k=0$ (dot-dashed line), $k=1$ (black line), $k=2$ (grey line), $k=3$ (dashed line), $k=4$ (dotted line)). Here $h=2$, but similar results are obtained with higher values of $c ; R_{0}$ is just smaller and $R_{o p t}$ is shifted to the left.

value of reserve size. On the other, someone wishing to postpone metapopulation extinction as long as possible will support maximizing reserve size, because this eventually increases the extinction time $T_{e}$. A strong argument of proponents of "the larger, the better" is that computing the exact value of the reserve size which optimizes $R_{0}$ may be a difficult task because it depends on species-specific parameters; miscalculations may have large consequences, especially when the calculated optimal reserve size is relatively small. Maximizing reserve size seems much safer in this respect.

However, the conclusion "the larger, the better" is possibly only valid for large $R$, because $T_{e}$ may have local optima at intermediate values of $R$. Of course, for any given area, there is a physical limit to the maximum reserve size that can be achieved. It is therefore possible that one would choose this maximum reserve size in a given area, but that, at least in theory, a smaller size has a larger extinction time. We remark that there is no direct relation between the specific values of $R$ where $R_{0}$ and $T_{e}$ achieve their optimum.

If $R_{0}$ is small enough such that the metapopulation is doomed for all $R$, there is a third possible opinion, which states that there should be a larger amount of habitable area to be distributed, because the main problem in this case is habitat loss, not habitat fragmentation. Habitat fragmentation becomes the basic problem once $R_{0}$ ceases to be so small, that is, when a viable metapopulation is theoretically possible. 
If the choice of the metapopulation persistence measure leads to disagreement even for a single species, one can expect even more discord when decisions influencing several species simultaneously need to be taken, especially when these species interact either directly or indirectly, for instance in a predator-prey system, or when a species is suffering from an infectious disease. In those, cases habitat fragmentation may have additional effects which are difficult, if not impossible, to handle under the assumptions made here (see e.g. Hassell et al., 1991; Hess, 1996).

This discussion is only warranted of course if one accepts the basic reproduction ratio $R_{0}$ as a measure of persistence. $R_{0}$ has been found to be a useful measure of infectious disease persistence. In that context it is interpreted as the number of individuals which are infected by a single infectious host. It has been shown that there is the following threshold behavior: if $R_{0}>1$ then the disease persists, if $R_{0}<1$ then it cannot persist (Diekmann, 1993; Diekmann et al., 1990). An important assumption for this threshold behavior at $R_{0}=1$ is that in the invasion phase, when the number of infected individuals is small, infectious particles do not fall on already infected hosts. If hosts are well mixed (i.e. they move around), this assumption can be reasonable for many infectious agents. Now, it seems natural to use $R_{0}$ and its threshold behavior at $R_{0}=1$ in a metapopulation context where patches take the role of hosts and dispersers take the role of spreading infectious particles. The analogous assumption for the threshold behavior at $R_{0}=1$ is then that in the invasion phase, when the patch occupation level is low, dispersers do not arrive in patches that are already occupied. Indeed, there are metapopulation models where this assumption is made, such as the Levins (1969) model, where all patches are supposed to be equally accessible. However, in the present setting, patches are fixed in a lattice and dispersal is distance-dependent, so patches are not equally accessible at all; even in a virgin environment (i.e. all patches except one are empty) dispersers will almost immediately settle in patches which are already occupied. Hence, the criterion $R_{0}>1$ no longer guarantees metapopulation persistence. This criterion would only be valid in the thought experiment in which patches are immediately replaced by a new empty patch every time a disperser arrives. So, the criterion now becomes $R_{0}>b$ for a certain $b>1$. It is, however, an unsolved mathematical problem to determine analytically the value of $b$ for the fixed lattice structure discussed in this paper. For the related contact process with nearest-neighbor dispersal in a square lattice, upper and lower bounds for $b$ have been found analytically (Durrett, 1992; Durrett \& Levins, 1994), while numerical studies give $b \approx 1.65$ when $T_{e}^{\text {local }}=1$ (Brower et al., 1978; Mollison, 1986) and $b \approx 2.13$ when $c=1$ (Durrett \& Levins, 1994). This shows that $b$ depends on the spatial configuration (which determines $T_{e}^{\text {local }}$ and $c$ ). Hence, $b$ may differ between the FL and MS cases! Thus, the combination of $R_{0}$ and $b$ instead of $R_{0}$ by itself needs to be specified to act as a persistence measure, and as the numerical examples above show, this can be done in practical applications.

Nevertheless, there are several reasons why $R_{0}$ by itself is worth considering as a persistence measure.

First of all, the condition for deterministic metapopulation extinction,

$$
R_{0}<1
$$

still holds, because.if less than one disperser sent out by a patch eventually settles in a patch, the metapopulation goes extinct. That is, eqn (36) is a sufficient, but no longer a necessary condition for metapopulation extinction.

Secondly, the overestimation of persistence may not be as severe as it looks. In the case of an infectious disease, every infectious particle falling on an already infected host, is completely wasted; it will not contribute to the persistence of the disease. But in the metapopulation case, dispersers arriving in an already occupied patch can still serve to increase metapopulation persistence by contributing to the local dynamics. In particular, they facilitate the rescue of local populations on the brink of extinction (the rescue effect, Brown \& Kodric-Brown, 1977; Hanski, 1983). In this respect, occupied patches act in a way as if they were empty, and the assumption for the threshold behavior at $R_{0}=1$ is not as severely violated as it seemed at first. 
Thirdly, this contribution to local dynamics in the fixed lattice can also be an advantage in the following sense. Suppose that dispersers always arrive in empty patches, thereby satisfying the assumption for the threshold behavior at $R_{0}=1$. Then at $R_{0}>1$ it may still not lead to metapopulation persistence if there is an Allee effect, that is, if more than one disperser is needed to start off a new local population. On the other hand, in the fixed lattice structure with distancedependent dispersal the Allee effect will be overcome quickly.

The results of this paper were derived for the most minimal setting in which the FLOMS problem makes sense. It remains to show that the results are qualitatively robust to relaxing the assumptions of this minimal setting. We will discuss the most prominent of them.

\section{THE MECHANISMS FOR $T_{e}^{\text {local }}(R), c_{\text {out }}(R)$ AND $c_{i n}(R)$}

The mechanisms for $T_{e}^{\text {local }}(R), c_{\text {out }}(R)$ and $c_{\text {in }}(R)$ may be inadequate, especially the ones describing $c_{\text {in. }}$ It is evident that the exponential part of $c_{\text {in }}(R)$ for large $\lambda_{1} R h$ plays a crucial role in the existence of an intermediate optimal reserve size. If Hill et al. (1996) are right about a power function instead of an exponential function, then there is no finite non-zero optimal reserve size. Therefore, more research is needed to enable models of dispersal and patch selection strategies for different classes of organisms (such as small mammals, birds, butterflies, and plants) that more closely adhere to what these organisms actually do when dispersing.

The power-law behavior of $T_{e}^{\text {local }}(R)$ for environmental stochasticity as opposed to exponential increase is the other crucial factor for the existence of an optimal reserve size. However, this behavior is not likely to change if local extinction were modeled in more detail.

As stated before, we used the (in our opinion) most minimal setting in which the FLOMS problem makes sense. This minimal setting comprises only the basics of the metapopulation concept for a single species: local extinction through demographic and environmental stochasticity, and recolonization driven by straight or random dispersal with a mortality risk. Of course, as mentioned previously, there are many more mechanisms by which fragmentation may be harmful or beneficial to metapopulation persistence. These mechanisms not only include singlespecies effects but also multiple-species effects, such as on the one hand increased local extinction risk because of the Allee effect, edge effects, minimum territory size, loss of key species in a food web and destabilization of mutualistic interactions, and on the other hand reduction of epidemic diseases, risk spreading in stochastic environments (bet hedging), increased genetic diversity, and the emergence of refugia from predators and competitors (Burkey, 1996; Verboom et al., 1993). These complicated mechanisms, which cannot be treated easily under the assumptions of this paper, may lead to different conclusions about the optimal size and number of reserves for a metapopulation, and deserve therefore to be studied. The outcome of this paper may serve as a null hypothesis for such further studies.

\section{VARIANCE IN PATCH SIZE AND PATCH DISTRIBUTION}

The analysis in this paper does not take variance in patch size and the spatial distribution of patches into account. Variance in patch size was found by Day \& Possingham (1995) to be of importance to the metapopulation extinction probability. They compared a scenario involving variable patch size with a scenario assuming equal patch size, where the total area of the eight patches under study is the same in both scenarios. For low colonization rates, systems with variable patch size are least extinction prone, whereas for high colonization rates equal patch size provides the longest expected lifetime of the metapopulation. Variance in patch distribution has been studied by Adler \& Nuernberger (1994), who reported a significant effect of this spatial distribution on metapopulation persistence. Day \& Possingham (1995) found only minor effects, but their study was not as extensive as Adler $\&$ Nuenberger's study and they used a different model.

What could be done in the present setup to incorporate variance in patch size is to first restate the FLOMS problem by comparing few patches with a large mean patch size and many 
patches with a small mean patch size, and then to check the behavior of the mean $\bar{R}_{0}(\bar{R})$ where $\bar{R}$ is the mean patch size. The problem is what mean to choose for $\bar{R}_{0}$. Possibly, ideas from Gyllenberg \& Hanski (1997), who study $R_{0}$ in a Levins-type metapopulation model with patches of different sizes, can be used for this purpose.

As far as patch distribution in space is concerned, the analysis in this paper, notably the formulation of the colonization potential which is representative of the entire metapopulation, cannot be extended in a simple way to cover patch configurations other than a regular lattice. It seems impossible to foretell whether the results will be similar or different from the regular pattern.

\section{INFLUENCE OF DISPERSAL ON EXTINCTION}

The assumption of the absence of the influence of dispersal on local population size and thereby on extinction is too crude. Gyllenberg \& Hanski $(1992,1997)$ have modeled the influence of dispersal on local population size mechanistically. However, the exercise of this paper cannot be repeated for their model because their model explicitly assumes a disperser pool which cannot be made consistent with distance-dependent dispersal (where distance in turn depends on $R)$. Models which incorporate both patchsize-dependent local dynamics and distancedependent dispersal are needed.

The connection between colonization and extinction may also be due to a different, although related, process. As Woodroffe \& Ginsberg (1998) note, patch (edge) size rather than population size determines the dispersal rate, making the patch boundaries (and hence small patches) into population sinks, i.e. $\alpha \leqslant 0$. In this case, Foley's models for the extinction rate prescribe behavior according to eqn (11) rather than eqn (12), but this does not change the qualitative dependence of $R_{o}$ and $T_{e}$ on $R$.

\section{SPATIAL CORRELATION OF LOCAL EXTINCTION TIMES}

The influence of spatial correlation of local extinction times on metapopulation persistence has been studied, but the literature suggests that it can go either way. For instance, using a Markovian metapopulation model with a finite number of patches, Akçakaya \& Ginzburg (1991) state that "Three Small" may be better than "Single Large" if spatial correlation is not too large. This agrees with the results of Frank \& Wissel (1998) who, using a model similar to Akçakaya \& Ginzburg, conclude that the metapopulation extinction time is significantly larger than local extinction time only if the dispersal distance exceeds the correlation length.

In this paper, the total habitable area was taken to be constant; changing patch size meant changing patch density simultaneously. This is of course the essence of the FLOMS problem. But the question is whether this problem is relevant, and hence whether the results of this paper are in any way applicable. If one can start from scratch to develop a given (fixed) amount of natural area, FLOMS is indeed the relevant problem, waiving the fact that there are more degrees of freedom for refuge design than just reserve size, such as reserve shape and spatial pattern (see e.g. Wilson $\&$ Willis, 1975). In practice, however, nature is already present (although declining). Decisions need to be made about where to develop nature (often in exchange for the destruction of habitat elsewehere in favor of roads or buildings), about where to put a corridor, or just about what to do about habitat fragmentation and habitat loss in general. The first issue is predominantly a FLOMS problem, but the second and third may involve much more, one reason being that habitat loss and habitat fragmentation often occur together. It would be advantageous to distinguish the effects of habitat fragmentation from those of habitat loss in a process that involves both, perhaps along the lines of Bascompte \& Sole (1996). But this is beyond the scope of this paper which just aimed to provide a view on the FLOMS problem in a minimal setting.

And all in all, most of the arguments above about the consequences of relaxing the assumptions in this minimal setting contribute to the robustness of the qualitative results that $R_{0}$ often shows a global optimum, whereas $T_{e}$ usually does not. Until empirical data or refined models (disperser behavior in particular needs looking into) convincingly show otherwise, one must be very careful in choosing few large instead of many small patches, but also in interpreting this warning as a license to fragment habitat. 
We are very grateful to Jana Verboom, Frank van den Bosch, Johan Grasman, Hans Metz and an anonymous referee for their helpful suggestions, references and discussions.

\section{REFERENCES}

Adler, F. R. \& Nuernberger, B. (1994). Persistence in patchy irregular landscapes. Theor. Pop. Biol. 45, 41-75.

AKCAKAYA, H. R. \& GinZBURG, L. R. (1991). Ecological risk analysis for single and multiple populations. In: Species Conservation: A Population-Biological Approach (Seitz, A. \& Loeschcke, V., eds), pp. 73-85. Basel, Switzerland: Birkhäuser Verlag.

Allen, E. J., Harris, J. M. \& Allen, L. J. S. (1992). Persistence-time models for use in viability analyses of vanishing species. J. theor. Biol. 155, 33-53.

BASCOMPTE, J. \& SolE, R. V. (1996). Habitat fragmentation and extinction thresholds in spatially explicit models. J. Anim. Ecol. 65, 465-473.

BAZ, A. \& GARCIA-BOYERO, A. (1996). The SLOSS dilemma: A butterfly case study. Biodiver. Conserv. 5, 493-502.

BeLL, W. J. (1991). Searching Behaviour: the Behavioural Ecology of Finding Resources. London: Chapman \& Hall. BoeCKLEN, W. J. (1986). Optimal design of nature reserves: consequences of genetic drift. Biol. Conserv. 38, 323-328.

Brower, R. C., Furman, M. A. \& Moshe, M. (1978). Critical exponents for the Reggeon quantum spin model. Phys. Lett. 76, 213-219.

Brown, J. H. \& Kodric-Brown, A. (1977). Turnover rate in insular biogeography: effect of immigration on extinction. Ecology 58, 445-449.

BURKEY, T. V. (1996). Sloss revisited - what do we really know about the effects of habitat fragmentation on extinction risk. In: Extinction in Fragmented Landscapes: Demographic Mechanisms and Predator-Prey Interactions (Ph.D. Thesis of Burkey, T.V.), pp. 172-225. Ann Arbor, MI: UMI. Dissertation Services.

Carslaw, H. S. \& Jaeger, J. C. (1959). Conduction of Heat in Solids. Oxford: Clarendon Press.

CrANK, J. (1975). The Mathematics of Diffusion. Oxford: Clarendon Press (1st Ed. 1956).

DAY, J. R. \& Possingham, H. P. (1995). A stochastic metapopulation model with variability in patch size and position. Theor. Pop. Biol. 48, 333-360.

DiEkMANN, O. (1993). An invitation to structured metapopulation models. In: Patch Dynamics (Levin, S. A., Powell, T. M. \& Steele, J. H., eds), pp. 162-175. Berlin: Springer-Verlag.

Diekmann, O., Heesterbeek, J. A. P. \& Metz, J. A. J. (1990). On the definition of the basic reproduction ratio $R_{0}$ in models for infectious diseases in heterogeneous populations. J. Math. Biol. 28, 365-382.

DURRETT, R. (1992). Stochastic growth models: bounds on critical values. J. Appl. Prob. 29, 11-20.

DurRetT, R. \& LeVINS, S. A. (1994). Stochastic spatial models: a user's guide to ecological applications. Phil. Trans. R. Soc. Lond. Ser. B 343, 329-350.

FAHRIG, L. (1992). Relative importance of spatial and temporal scales in a patchy environment. Theor. Popul. Biol. 41, 300-314.
FOLEY, P. (1997). Extinction models for local populations. In: Metapopulation Biology. Ecology, Genetics, and Evolution (Hanski, I. A. \& Gilpin, M. E., eds), pp. 215-246. San Diego: Academic Press.

ForNEY, K. A. \& GiLPIN, M. E. (1989). Spatial structure and population extinction: a study with Drosophila flies. Conserv. Biol. 3, 45-51.

FrANK, K. \& Wissel, C. (1998). Spatial aspects of metapopulation survival - from model results to rules of thumb for landscape management. Landscape Ecol. 13, 363-379.

FrankHAM, R. \& RALLS, K. (1998). Inbreeding leads to extinction. Nature 392, 441-442.

Gilpin, M. E. \& DiAmond, J. M. (1976). Calculation of immigration and extinction curves from the speciesarea-distance relation. Proc. Nat. Acad. Sci. U.S.A. 73, 4130-4134.

Gonzalez, A., Lawton, J. H., Gilbert, F. S., Blackburn, T. M. \& EvANS-FreKE, I. (1998). Metapopulation dynamics: abundance, and distribution in a microecosystem. Science 281, 2045-2047.

Goodman, D. (1987). The demography of chance extinction. In: Viable Populations (Soulé, M. E., ed.), pp. 11-34. Cambridge: Cambridge University Press.

GyllenberG, M. \& Hanski, I. A. (1992). Single-species metapopulation dynamics: a structured model. Theor. Popul. Biol. 42, 35-61.

GyllenberG, M. \& HANSKI, I. A. (1997). Habitat deterioration, habitat destruction, and metapopulation persistence in a heterogenous landscape. Theor. Popul. Biol. 52, 198-215.

HANSKI, I. A. (1983). Coexistence of competitors in patchy environment. Ecology 64, 493-500.

HANSKI, I .A. (1991). Single-species metapopulation dynamics: concepts, models and observations. Biol. J. Linnean Soc. 42, 17-38.

HANSKI, I. A. (1992). Inferences from ecological incidence functions. Am. Nat. 139, 657-662.

HANSKI, I. A. (1997). Metapopulation dynamics: from concepts and observations to predictive models. In: Metapopulation Biology. Ecology, Genetics, and Evolution (Hanski, I. A. \& Gilpin, M. E., eds), pp. 69-91. San Diego: Academic Press.

Hanski, I. A., Moilanen, A., Pakkala, T. \& KuUssaari, M. (1996). The quantitative incidence function model and persistence of an endangered butterfly metapopulation. Conserv. Biol. 10, 578-590.

HANSSON, L. (1991). Dispersal and connectivity in metapopulations. Biol. J. Linnean Soc. 42, 89-103.

Hassell, M. P., Comins, H. N. \& MAY, R. M. (1991). Spatial structure and chaos in insect population dynamics. Nature 353, 255-258.

Hess, G. (1996). Disease in metapopulation models: implications for conservation. Ecology 77, 1617-1632.

Hill, J. K., Thomas, C. D. \& Lewis, O. T. (1996). Effects of habitat patch size and isolation on dispersal by Hesperia comma butterflies: implications for metapopulation structure. J. Anim. Ecol. 65, 725-735.

Kindvall, O. \& Ahlen, I. (1992). Geometrical factors and metapopulation dynamics of the Bush Cricket, Metrioptera bicolor Philippi (Orthoptera: Tettigoniidae). Conserv. Biol. 6, 520-529.

LEVINS, R. (1969). Some demographic and genetic consequences of environmental heterogeneity for biological control. Bull. Entomol. Soc. Am. 15, 237-240. 
Lindenmayer, D. B. \& Possingham, H. P. (1995). Modelling the viability of metapopulations of the endangered Leadbeater's Possum in South-Eastern Australia. Biodiv. Conserv. 4, 984-1018.

MacArthur, R. H. \& Wilson, E. O. (1967). Island Biogeography. Princeton: Princeton University Press.

Manne, L. L., Pimm, S. L., Diamond, J. M. \& Reed, T. M. (1998). The form of the curves: a direct evaluation of MacArthur \& Wilson's classical theory. J. Anim. Ecol. 67, 784-794.

Mollison, D. (1986). Modelling biological invasions: chance, explanation, prediction. Phil. Trans. R. Soci. Lond. B 314, 675-693.

POOLER, J. (1987). Measuring geographical accessibility: a review of current approaches and problems in the use of population potentials. Geoforum 18, 269-289.

Possingham, H. P. \& DAvies, I. (1995). ALEX: a population viability analysis model for spatially structured populations. Biol. Conserv. 73, 143-150.

Possingham, H. P., Davies, I., Noble, I. R. \& Norton, T. W. (1992). A metapopulation simulation model for assessing the likelihood of plant and animal extinctions. Math. Comput. Simulation 33, 367-372.
Wardle, D. A., Zackrisson, O., Hornberg, G. \& GalLET, C. (1997). The influence of island area on ecosystem properties. Science 277, 1296-1299.

Wilson, E. O. \& Willis, E. O. (1975). Applied biogeography. In: Ecology and Evolution of Communities (Cody, M. L. \& Diamond, J. M., eds), pp. 523-534. Cambridge, MA: Harvard University Press.

WoOdroffe, R. \& GinsBerG, J. R. (1998). Edge effects and the extinction of populations inside protected areas. Science 280, 2126-2128.

\section{APPENDIX A}

\section{Straight Walk and Exponential Decline outside Circular Patches in a Lattice}

For the settlement-death strategy with straight walk in a hexagonal $(n=6)$ or square $(n=4)$ lattice, eqns (17), (18) and (21) can be worked out to give

$$
c_{i n}(R, n)=\mathrm{e}^{(3 / 2) \lambda_{1} R} \frac{\sum_{k=1}^{\infty} \sum_{l=0}^{\infty} n \mathrm{e}^{-\left(\lambda_{1}+\lambda_{2}\right) R h \sqrt{k^{2}+l^{2}-2 k l \cos \pi(1-2 / n)}}}{\sum_{k=1}^{\infty} \sum_{l=0}^{\infty} n \mathrm{e}^{-\lambda_{2} R h \sqrt{k^{2}+l^{2}-2 k l \cos \pi(1-2 / n)}}}=: \mathrm{e}^{3 / 2 \lambda_{1} R} \frac{S_{1}\left(\lambda_{1}+\lambda_{2}\right)}{S_{1}\left(\lambda_{2}\right)} .
$$

Quinn, J. F. \& Harrison, S. P. (1988). Effects of habitat fragmentation and isolation on species richness: evidence from biogeographic patterns. Oecologia 75, $132-140$.

SACCheri, I., KuUssaAri, M., KANKARE, M., ViKMan, P., FORTELIUS, W. \& HANSKI, I. (1998). Inbreeding and extinction in a butterfly metapopulation. Nature 392, 491-494.

SCHAFFER, M. L. (1981). Minimum population sizes for species conservation. Biosciences 31, 131-134.

Spiller, D. A., Losos, J. B. \& Schoener, T. W. (1998). Impact of a catastrophic hurricane on island populations. Science 281, 695-697.

Stacey, P. B., Johnson, V. A. \& TAper, M. L. (1997). Migration within metapopulations. The impact upon local population dynamics. In: Metapopulation Biology. Ecology, Genetics, and Evolution (Hanski, I. A. \& Gilpin, M. E., eds), pp. 267-291. San Diego: Academic Press.

Verboom, J., Metz, J. A. J. \& Meelis, E. (1993). Metapopulation models for impact assessment of fragmentation. In: Landscape Ecology of a Stressed Environment. IALE Studies in Landscape Ecology 1 (Vos, C. C. \& Opdam, P., eds), pp. 173-191. London: Chapman \& Hall.

Vos, C. C., Verboom, J., Opdam, P. F. M., Ter BraAk, C. J. F., Bergers, P. J. M. \& VAN APELdoORN, R. C. (1999). Towards ecologically scaled landscape indices. In: A Frog's-Eye View of the Landscape. Quantifying Connectivity for Fragmented Amphibian Populations (Ph.D. Thesis of Vos, C. C.), pp. 92-115. Wageningen, The Netherlands: Wageningen University.
Note that $h$, the constant representing the density of habitable area, must be greater than $3 / 2$; this is always satisfied, since the patches should not overlap which entails $h>2$ for $n=4$ and $h>12^{1 / 4} \approx 2$ for $n=6$. Approximation of the double sum $S_{1}(x)$ in eqn (A.1) by its first term leads to

$$
S_{1}(x) \approx n \mathrm{e}^{-x R h} \text { for large } x R h
$$

which is a lower boundary of $S_{1}$. Approximation of $S_{1}(x)$ by the corresponding integral gives

$$
S_{1}(x) \approx \frac{2 \pi}{(x R h)^{2} \sin 2 \pi / n} \text { for small } x R h
$$

which is an upper boundary of $S_{1}$. See Fig. A1 for an impression of the accuracy of these approximations. With eqns (A.2) and (A.3), $\quad c_{i n}(R, n)$ can be approximated 

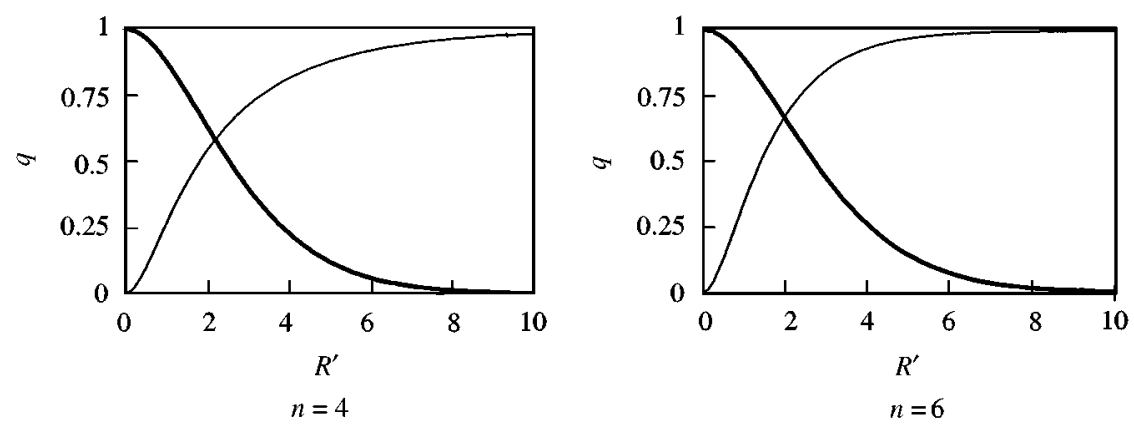

FIG. A1. Plots of approximations (A.2) and (A.3) to $S_{1}$ as functions of the rescaled patch radius $R^{\prime}=x R h$ for $n=4$ (left) and $n=6$ (right). The approximations are plotted as quotients $q$ relative to $S_{1} ;(-): q\left(R^{\prime}\right)=$ eqn $(\mathrm{A} .2) / S_{1}\left(R^{\prime}\right)$, $(-): q\left(R^{\prime}\right)=S_{1}\left(R^{\prime}\right) /$ eqn (A.3). $S_{1}$ is defined in eqn (A.1).

by

$$
c_{i n}(R, n)= \begin{cases}\mathrm{e}^{(3 / 2) \lambda_{1} R}\left(\frac{\lambda_{2}}{\lambda_{1}+\lambda_{2}}\right)^{2} & \text { for }\left(\lambda_{1}+\lambda_{2}\right) R h \ll 1 \\ \frac{n\left(\lambda_{2} R h\right)^{2} \sin (2 \pi / n)}{2 \pi} \mathrm{e}^{-\lambda_{1} R(h-(3 / 2))-\lambda_{2} R h} & \text { for } \lambda_{1} R h \gg 1 \text { and } \lambda_{2} R h \ll 1\left(\text { so } \lambda_{2} / \lambda_{1} \ll 1\right), \\ \mathrm{e}^{-\lambda_{1} R(h-(3 / 2))} & \text { for } \lambda_{2} R h \gg 1 .\end{cases}
$$

For the pie-slice strategy with straight walk, simple geometry teaches that $\Delta \varphi_{i}(R, n)$ is given by

$$
\Delta \varphi_{i}(R, n)=2 \arccos \left[1-\frac{1}{2 h^{2} u_{i}^{2}}\right]
$$

where $u_{i}:=d_{i} / L$ is the distance from patch center to patch center expressed in units of the lattice distance $L$. Note that $\Delta \varphi_{i}(R, n) \equiv \Delta \varphi_{i}$ is independent of the size of the patches.

The fraction $f_{i}$ depends on whether there are patches in front of patch $i$, blocking the view from the patch of origin. To facilitate straightforward calculation, we assume that
The unobservable patches are easily found, because the quotient of their coordinates are either the same as that of a closer patch, or $\sum_{j=0}^{m} \Delta \varphi_{j} \geqslant 2 \pi$ for a patch $m$ which is closer than they are.

The survival probability $p_{\text {survival }}(R)$ can be written in terms of $u_{i}$ as well:

$$
p_{\text {survival }}(R)=\mathrm{e}^{-\lambda_{1}\left(d_{i}-(3 / 2) R\right)}=\mathrm{e}^{-\lambda R\left(u_{i} h-(3 / 2)\right)}
$$

Thus,

$$
\begin{aligned}
c_{i n}(R, n)= & \mathrm{e}^{3 / 2 \lambda_{1} R} \sum_{i} \frac{f_{i}}{\pi} \mathrm{e}^{-\lambda_{1} R u_{i} h} \\
& \times \arccos \left[1-\frac{1}{2 h^{2} u_{i}^{2}}\right]=: \mathrm{e}^{3 / 2 \lambda_{1} R} S_{2} .
\end{aligned}
$$



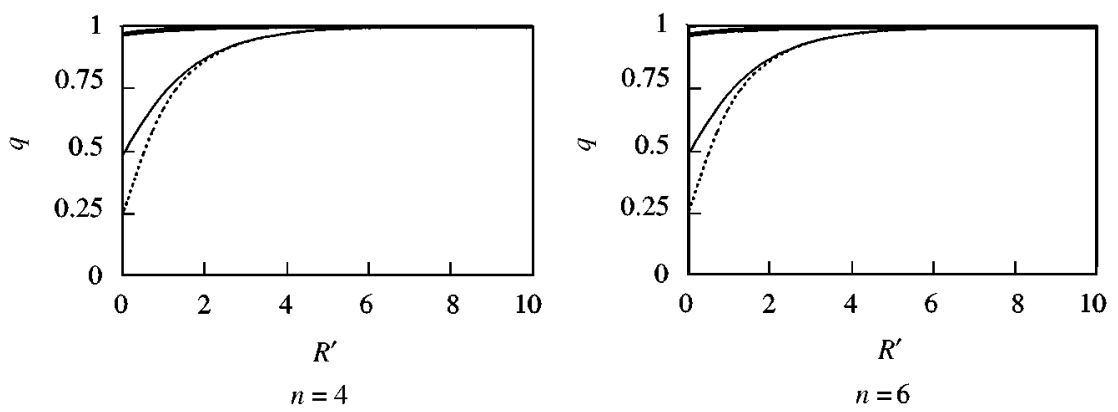

FIG. A2. Plots of the approximation (A.9) to $S_{2}$ as a function of the rescaled patch radius $R^{\prime}=\lambda_{1} R h$ for $n=4$ (left) and $n=6$ (right). The approximation is plotted as a quotient $q$ relative to $S_{2}$ for three values of $h$, that is $q=$ eqn (A.9)/ $S_{2}\left(R^{\prime}\right) . S_{2}$ is defined in eqn (A.8). $\left.(\cdots)\right) h=8 ;(-) h=4 ;(-) h=2$.

A fairly good approximation to the sum $S_{2}$ in eqn (A.8) is its first term for which all $f_{i}$ and $u_{i}$ are 1 :

$$
S_{2} \approx \frac{n}{\pi} \mathrm{e}^{-\lambda_{1} R h} \arccos \left[1-\frac{1}{2 h^{2}}\right] \text { for large } \lambda_{1} R h
$$

as can be seen from Fig. A2. No such simple approximation is possible for small $\lambda_{1} R h$ because of the dependence of the arccos term on $h$, which is also evident from Fig. A2. With this approximation eqn (A.8) yields

$$
\begin{gathered}
c_{i n}(R, n) \approx \frac{n}{\pi} \mathrm{e}^{-\lambda_{1} R(h-3 / 2)} \arccos \left[1-\frac{1}{2 h^{2}}\right] \\
\text { for } \lambda_{1} R h \gg 1
\end{gathered}
$$

\section{APPENDIX B}

\section{Random Walk and Exponential Decline outside Circular Patches in a Lattice}

Here expressions are derived for $c_{i n}(R)$ using the stationary $(\partial x / \partial t=0, x$ is disperser density) diffusion equation (Carslaw \& Jaeger, 1959; Crank, 1975) with a constant net death rate $\lambda$ outside circular patches with radius $R$ and diffusion coefficient $D$,

$$
D\left(\frac{\partial^{2} x}{\partial r^{2}}+\frac{1}{r} \frac{\partial x}{\partial r}\right)-\lambda x=0
$$

subject to the boundary conditions

$$
\begin{gathered}
\lim _{r \rightarrow \infty} x(r)=0, \\
\left.\lim _{r^{\prime} \backslash R} D \frac{\partial x}{\partial r}\right|_{r=r^{\prime}}=c_{\text {out }}(R) .
\end{gathered}
$$

The solution is, defining $\lambda_{1}:=\sqrt{\lambda / D}$,

$$
x(r)= \begin{cases}C_{1}+C_{2} \ln r & \text { for } \lambda_{1}=0, \\ C_{3} I_{0}\left(\lambda_{1} r\right)+C_{4} K_{0}\left(\lambda_{1} r\right) & \text { for } \lambda_{1}>0,\end{cases}
$$

where $I_{j}$ and $K_{j}$ are the $j$-th-order modified Bessel functions of the first and second kind. The boundary condition (B.2) requires

$$
C_{1}=C_{2}=C_{3}=0
$$

leaving only the solution

$$
x(r)=C_{4} K_{0}\left(\lambda_{1} r\right)
$$

The second boundary condition (B.3) gives

$$
C_{4}=-\frac{c_{\text {out }}(R)}{D \lambda_{1} K_{1}\left(\lambda_{1} R\right)}
$$


For eqn (25) with the pie-slice strategy it follows that

$$
\begin{aligned}
c_{\text {in }}(R) & \approx \frac{-\left.\sum_{i} \lim _{r^{\prime} \uparrow d_{i}-(1 / 2) R} D \frac{\partial x}{\partial r}\right|_{r=r^{\prime}} f_{i}\left(d_{i}-\frac{1}{2} R\right) \Delta \varphi_{i}}{c_{\text {out }}(R)} \\
& =\sum_{i} \frac{D C_{4} \lambda_{1} K_{1}\left(\lambda_{1}\left(d_{i}-\frac{1}{2} R\right)\right)\left(d_{i}-\frac{1}{2} R\right) f_{i}}{c_{\text {out }}(R)} \arccos \left[1-\frac{1}{2 h^{2} u_{i}^{2}}\right] \\
& =\sum_{i} \frac{K_{1}\left(\lambda_{1}\left(d_{i}-\frac{1}{2} R\right)\right)\left(d_{i}-\frac{1}{2} R\right) f_{i}}{K_{1}\left(\lambda_{1} R\right)} \arccos \left[1-\frac{1}{2 h^{2} u_{i}^{2}}\right],
\end{aligned}
$$

where either $f_{i}=1$ for all $i$, or $f_{i}$ is given by eqn (A.6), and $\Delta \varphi_{i}$ and $C_{4}$ are given by eqns (A.5) and (B.7), respectively. The term $\frac{1}{2}$ is subtracted from $d_{i}$ to account for the fact that part of $d_{i}$ lies inside the patch of destination. For large $\lambda_{1} R h$, we can replace the sum in eqn (B.8) by its first term and approximate $K_{1}$ to get

$$
\begin{aligned}
& c_{i n}(R) \approx n R \sqrt{h-\frac{1}{2}} \mathrm{e}^{-\lambda_{1} R(h-3 / 2)} \\
& \times \arccos \left[1-\frac{1}{2 h^{2}}\right] \text { for large } \lambda_{1} R h
\end{aligned}
$$

